\title{
Existence, Uniqueness and Stability of Nonlinear Implicit Fractional Dynamical Equation with Impulsive condition on Time Scales
}

https://doi.org/10.1515/msds-2019-0005

Received October 31, 2018; accepted October 5, 2019

\begin{abstract}
The main motive of this research article is to establish the existence, uniqueness and stability results for the non-linear fractional differential equation with impulsive condition on time scales. Banach, Leray-Schauder's alternative type fixed point theorems are used to examine these results. Further, we give the existence and uniqueness of solution for the corresponding non-local problem. Moreover, to outline the utilization of these outcomes some examples are given.
\end{abstract}

Keywords: Time Scales, Existence, Stability, Fractional Impulsive Differential Equations, Fixed Point Theorem

MSC: 34N05, 34A08, 34A12, 03C45, 34A26

\section{Introduction}

In many physical problems of engineering and science the fractional differential equations are arise for e.g control problem, image processing, signal identification, optical systems and so on, see [1,2] and references therein. A lot of attention has been given by scholars to it and established the various results on existence and uniqueness of different type of fractional differential equations. As of late, numerous researchers paid much attention to the existence of initial and boundary value problem with fractional differential equations with different-different boundary conditions using the various type of fixed point theorems like contraction, Schaefer's, Schauder's, Krasnoselskii's and so on, see [3-10] and references therein.

There are many physical problems that are described by sudden changes in their states. These sudden changes are called the impulsive effects in the system. The theory of impulsive differential equations have found tremendous applications in engineering and science. It has arisen a significant area of research such as population dynamics, ecology, biotechnology, modeling of impulsive problems in the science of matter and so forth, see the monograph given by Lakshmikantham et al. [11], Benchohra et al. [12] and references therein.

These results have been studied in depth in both the continuous and discrete cases. In 1988, Hilger [13] presented the time scales calculus. The investigation of calculus on time scales encapsulates both the continuous as well as discrete analysis of the system, therefore the study of dynamical systems on time scales has gained a great attention and many researchers have found the applications of time scales in the heat

\footnotetext{
*Corresponding Author: Vipin Kumar: School of Basic Sciences, Indian Institute of Technology Mandi, H. P. India, E-mail: math.vipinkumar219@gmail.com

Muslim Malik: School of Basic Sciences, Indian Institute of Technology Mandi, H. P. India, E-mail:

E-mail: muslim@iitmandi.ac.in
} 
transfer system [14], population dynamics [15] and as well as in economics [16, 17]. For more details about time scales please see [18-21].

In the last few years, Vipin et al. [22], Ahmadkhanlu et al. [23], Benkhettou et al. [24], Yan et al. [25] and references therein, have been worked on fractional differential equations on time scales. Particularly, Ahmadkhanlu et al. [23], give the necessary and sufficient conditions for the existence and uniqueness of solution to the fractional order differential equations (FODE) on time scales:

$$
\begin{aligned}
{ }^{c} \Delta^{\alpha} z(\theta) & =\mathcal{F}(\theta, z(\theta)), \quad \forall \theta \in J=\left[\theta_{0}, \theta_{0}+a\right] \subseteq \mathbb{T}, \quad \alpha \in(0,1], \\
z\left(\theta_{0}\right) & =z_{0},
\end{aligned}
$$

where $\mathcal{F}: J \times \mathbb{R} \rightarrow \mathbb{R}$ is a right-dense continuous bounded function and ${ }^{c} \Delta^{\alpha}$ is the Caputo fractional derivative. In 2004, Agarwal et al. [26], used the Schaefer's fixed point theorem to establish the existence of solutions for an impulsive dynamic system of second order on time scales $\mathbb{T}$

$$
\begin{aligned}
& z^{\Delta \Delta}(\theta)=\mathcal{F}(\theta, z(\theta)), \quad \forall \theta \in J:=[0, b], \theta \neq \theta_{l}, l=1,2, \cdots, p, \\
& z\left(\theta_{l}^{+}\right)-z\left(\theta_{l}^{-}\right)=I_{l}\left(z\left(\theta_{l}^{-}\right)\right), l=1,2, \cdots, p, \\
& z^{\Delta}\left(\theta_{l}^{+}\right)-z^{\Delta}\left(\theta_{l}^{-}\right)=\bar{I}_{l}\left(z\left(\theta_{l}^{-}\right)\right), l=1,2, \cdots, p, \\
& \quad z(0)=z_{0}, \quad z^{\Delta}(0)=z_{1},
\end{aligned}
$$

with $0, b, z_{0}, z_{1} \in \mathbb{T}, \mathcal{F}(\theta, z(\theta))$ is a given function, $I_{l}, \bar{I}_{l} \in C(\mathbb{R}, \mathbb{R}) .0 \leq \theta_{0}<\theta_{1}<\theta_{2}<\cdots<\theta_{p}<\theta_{p+1}=T$, $z\left(\theta_{l}^{-}\right)=\lim _{h \rightarrow 0^{+}} z\left(\theta_{l}-h\right), z\left(\theta_{l}^{+}\right)=\lim _{h \rightarrow 0^{+}} z\left(\theta_{l}+h\right)$ denotes the left and right limits of $z(\theta)$ at $\theta=\theta_{l}$.

Another thing of the qualitative principle which could be very important from optimization and numerical point of view is dedicated to stability analysis of the solution to differential equations of integer as well as fractional order. In this regard, Hyers-Ulam kind stability for the solution of differential equations of integer as well as fractional order has been delivered in lots of articles. The concept of Hyers-Ulam type stability has been introduced in $19^{\text {th }}$ century and now it has been gained a lot of attention. There are many authors which discussed the Ulam's type stability for the ordinary as well as for the fractional differential and difference equations [27-29] and references therein. Moreover, many authors have been discussed these results on time scales [30, 31] and references therein.

As per our knowledge, there is no paper which discussed the existence, uniqueness and stability analysis for the nonlinear FODE with impulses on time scales. Spurred by the above facts, we consider the implicit nonlinear FODE with impulses on time scale of the form:

$$
\begin{aligned}
{ }^{c} \Delta^{q} w(\theta) & =\mathcal{G}\left(\theta, w(\theta),{ }^{c} \Delta^{q} w(\theta)\right), \quad \theta \in I=[0, T]_{\mathbb{T}}, \theta \neq \theta_{l}, \\
w\left(\theta_{l}^{+}\right) & -w\left(\theta_{l}^{-}\right)=\mathcal{J}_{l}\left(\theta_{l}, w\left(\theta_{l}^{-}\right)\right), \quad l=1,2, \cdots, p, \\
w(0) & =w_{0},
\end{aligned}
$$

where $\mathbb{T}$ is a time scale with $0, \theta_{l}, T \in \mathbb{T},{ }^{c} \Delta^{q}$ is the Caputo fractional derivative with $q \in(0,1)$. Throughout the paper, we assumed that for $l=1,2, \cdots, p$, the point of impulses $\theta_{l}$ are right dense with $0 \leq \theta_{0}<\theta_{1}<$ $\theta_{2}<\cdots<\theta_{p}<\theta_{p+1}=T . w\left(\theta_{l}^{-}\right)=\lim _{h \rightarrow 0^{+}} w\left(\theta_{l}-h\right), w\left(\theta_{l}^{+}\right)=\lim _{h \rightarrow 0^{+}} w\left(\theta_{l}+h\right)$ denote the left and the right limits of $w(\theta)$ at $\theta=\theta_{l}$. $\mathcal{J}_{l} \in C(I \times \mathbb{R}, \mathbb{R})$ and $\mathcal{G}: I \times \mathbb{R} \times \mathbb{R} \rightarrow \mathbb{R}$ are given functions.

The plan of the manuscript is as follows. In Section 2, we give some preliminaries, fundamental definitions and some useful lemmas. In Section 3, the main results of the paper are discussed. In Section 4, we give the existence and uniqueness of solution for the nonlocal problem corresponding to the equation (1.1). At last, to outcome the utilization of these obtained analytical results, two examples are given.

\section{Preliminaries}

In this section, we briefly described basic notations, fundamental definitions and useful lemmas. $C(I, \mathbb{R})$ and $P C(I, \mathbb{R})$ represents the space of all continuous and peace wise continuous functions respectively where 
$P C(I, \mathbb{R})=\left\{w: I \rightarrow \mathbb{R}: w \in C\left(\left(\theta_{l}, \theta_{l+1}\right]_{\mathbb{T}}, \mathbb{R}\right), l=0,1, \cdots, p\right.$ and there exist $w\left(\theta_{l}^{-}\right)$and $w\left(\theta_{l}^{+}\right), \quad l=$ $1,2, \cdots, p$ with $\left.w\left(\theta_{l}^{-}\right)=w\left(\theta_{l}\right)\right\}$. one can easily find that $P C(I, \mathbb{R})$ for all $\theta \in I$, is a Banach space with the norm,

$$
\|\Psi\|_{P C}:=\sup \{|\Psi(\zeta)|, \quad 0 \leq \zeta \leq \theta\} .
$$

Further, we define $P C^{1}(I, \mathbb{R})=\left\{w \in P C(I, \mathbb{R}): w^{\Delta} \in P C(I, \mathbb{R})\right\} . P C^{1}(I, \mathbb{R})$ form a Banach space endowed with the norm $\|w\|_{1}=\max \left\{\|w\|_{P C},\left\|w^{\Delta}\right\|_{P C}\right\}$.

A closed non-empty subset of real number is called time scales $\mathbb{T}$. We define a time scale interval as $[a, b]_{\mathbb{T}}=\{\theta \in \mathbb{T}: a \leq \theta \leq b\}$, accordingly, we define $(a, b)_{\mathbb{T}},[a, b)_{\mathbb{T}},(a, b]_{\mathbb{T}}$ and so on. Also, we define $\mathbb{T}^{k}=\mathbb{T} \backslash\{\max \mathbb{T}\}$ if $\max \mathbb{T}$ exists, otherwise $\mathbb{T}^{k}=\mathbb{T}$. The forward jump operator $\sigma: \mathbb{T} \rightarrow \mathbb{T}$ is defined by $\sigma(\theta):=\inf \{t \in \mathbb{T}: t>\theta\}$ with the substitution $\inf \{\phi\}=\sup \mathbb{T}$ and the graininess function $\mu: \mathbb{T} \rightarrow[0, \infty)$ by $\mu(\theta):=\sigma(\theta)-\theta, \forall \theta \in \mathbb{T}$.

Definition 2.1. [18] Let $r: \mathbb{T} \rightarrow \mathbb{R}$ and $\theta \in \mathbb{T}^{k}$. The delta derivative $r^{\Delta}(\theta)$ is the number (when it exists) such that given any $\epsilon>0$, there is a neighbourhood $U$ of $\theta$ such that

$$
\left|[r(\sigma(\theta))-r(\tau)]-r^{\Delta}(\theta)[\sigma(\theta)-\tau]\right| \leq \epsilon|\sigma(\theta)-\tau|, \quad \forall \tau \in U .
$$

Definition 2.2. [18] Let $R$ be a function, it is called the antiderivative of $r: \mathbb{T} \rightarrow \mathbb{R}$ provided $R^{\Delta}(\theta)=r(\theta)$ for each $\theta \in \mathbb{T}^{k}$, then the delta integral is defined by

$$
\int_{\theta_{0}}^{\theta} r(\zeta) \Delta \zeta=R(\theta)-R\left(\theta_{0}\right) .
$$

A function $r: \mathbb{T} \rightarrow \mathbb{R}$ is said to be rd-continuous on $\mathbb{T}$, if $r$ has finite left-sided limits at points $\theta \in \mathbb{T}$ with $\sup \{t \in \mathbb{T}: t<\theta\}=\theta$ and $r$ is continuous at points $\theta \in \mathbb{T}$ with $\sigma(\theta)=\theta$. The collections of all rd-continuous functions $r: \mathbb{T} \rightarrow \mathbb{R}$ will be denoted by $C_{r d}(\mathbb{T}, \mathbb{R})$.

Theorem 2.3. [21] Let $r: \mathbb{R} \rightarrow \mathbb{R}$ be a continuous, nondecreasing function, and let $\mathbb{T}$ be an arbitrary time scale with $\theta_{1}, \theta_{2} \in \mathbb{T}$, such that $\theta_{1} \leq \theta_{2}$ then,

$$
\int_{\theta_{1}}^{\theta_{2}} r(\zeta) \Delta \zeta \leq \int_{\theta_{1}}^{\theta_{2}} r(\zeta) d \zeta
$$

Definition 2.4. [22] Let $r:[a, b]_{\mathbb{T}} \rightarrow \mathbb{R}$ is an integrable function, then $\Delta$-fractional integral of $r$ is given by

$$
{ }^{\Delta} I_{a^{+}}^{q} r(\theta)=\int_{a}^{\theta} \frac{(\theta-\zeta)^{q-1}}{\Gamma(q)} r(\zeta) \Delta \zeta,
$$

where $\Gamma(q)$ denotes the usual Euler Gamma function.

Definition 2.5. [22] Let $r: \mathbb{T} \rightarrow \mathbb{R}$. The Caputo $\Delta$-fractional derivative of $r$ is defined by

$$
{ }^{c} \Delta_{a^{+}}^{q} r(\theta)=\int_{a}^{\theta} \frac{(\theta-\zeta)^{n-q-1}}{\Gamma(n-q)} r^{\Delta^{n}}(\zeta) \Delta \zeta,
$$

where $n=[q]+1$ and $[q]$ denotes the integer part of $q$.

Lemma 2.6. [23] If $r$ is a integrable function on $[a, b]_{\mathbb{T}}$, then the $\Delta$-fractional integral satisfies ${ }^{\Delta} I_{a^{+}}^{q_{1} \Delta} I_{a^{+}}^{q_{2}} r={ }^{\Delta}$ $I_{a^{+}}^{q_{1}+q_{2}} r$.

Theorem 2.7. [23] Let $q \in(0,1)$ and $\mathcal{F}: I \times \mathbb{R} \rightarrow \mathbb{R}$ be a given function then the function $w(\theta)$ is a solution of

$$
{ }^{c} \Delta^{q} w(\theta)=\mathcal{F}(\theta, w(\theta)), w(0)=w_{0},
$$


if and only if $w(\theta)$ is the solution of the following integral equation :

$$
w(\theta)=w_{0}+\frac{1}{\Gamma(q)} \int_{0}^{\theta}(\theta-\zeta)^{q-1} \mathcal{F}(\zeta, w(\zeta)) \Delta \zeta
$$

Definition 2.8. A function $w(\theta) \in P C(I, \mathbb{R})$ is called a solution of equation (1.1), if for each $l=1,2, \cdots, p, w$ satisfies the equation ${ }^{c} \Delta^{q} w(\theta)=\mathcal{G}\left(\theta, w(\theta),{ }^{c} \Delta^{q} w(\theta)\right)$ a.e. on I and the conditions $w\left(\theta_{l}^{+}\right)-w\left(\theta_{l}^{-}\right)=\mathcal{J}_{l}\left(\theta_{l}, w\left(\theta_{l}^{-}\right)\right)$, $w(0)=w_{0}$.

Lemma 2.9. Let $g: I \rightarrow \mathbb{R}$ be a right dense continuous function. Then for any $l=1,2, \cdots, p$, the solution of the following problem

$$
\begin{aligned}
{ }^{c} \Delta^{q} w(\theta) & =g(\theta), \quad \theta \in I, \quad \theta \neq \theta_{l}, \\
w\left(\theta_{l}^{+}\right) & -w\left(\theta_{l}^{-}\right)=\mathcal{J}_{l}\left(\theta_{l}, w\left(\theta_{l}^{-}\right)\right), \quad l=1,2, \cdots, p, \\
w(0) & =w_{0},
\end{aligned}
$$

is given by the following integral equation

$$
w(\theta)= \begin{cases}w_{0}+\frac{1}{\Gamma(q)} \int_{0}^{\theta}(\theta-\zeta)^{q-1} g(\zeta) \Delta \zeta, & \theta \in\left[0, \theta_{1}\right]_{\mathbb{T}} . \\ w_{0}+\frac{1}{\Gamma(q)} \sum_{i=1}^{l} \int_{\theta_{i-1}}^{\theta_{i}}\left(\theta_{i}-\zeta\right)^{q-1} g(\zeta) \Delta \zeta+\frac{1}{\Gamma(q)} \int_{\theta_{l}}^{\theta}(\theta-\zeta)^{q-1} g(\zeta) \Delta \zeta & \\ \quad+\sum_{i=1}^{l} \partial_{i}\left(\theta_{i}, w\left(\theta_{i}^{-}\right)\right), & \theta \in\left(\theta_{l}, \theta_{l+1}\right]_{\mathbb{T}} .\end{cases}
$$

Proof: If $\theta \in\left[0, \theta_{1}\right]_{\mathbb{T}}$, then the solution of equation (2.4) is given by

$$
w(\theta)=w_{0}+\frac{1}{\Gamma(q)} \int_{0}^{\theta}(\theta-\zeta)^{q-1} g(\zeta) \Delta \zeta .
$$

For $\theta \in\left(\theta_{1}, \theta_{2}\right]_{\mathbb{T}}$, the initial value problem

$$
\left\{\begin{array}{l}
{ }^{c} \Delta^{q} w(\theta)=g(\theta), \\
w\left(\theta_{1}^{+}\right)-w\left(\theta_{1}^{-}\right)=\mathcal{J}_{1}\left(\theta_{1}, w\left(\theta_{1}^{-}\right)\right),
\end{array}\right.
$$

has the solution

$$
w(\theta)=w\left(\theta_{1}^{+}\right)+\frac{1}{\Gamma(q)} \int_{\theta_{1}}^{\theta}(\theta-\zeta)^{q-1} g(\zeta) \Delta \zeta
$$

It follows that

$$
\begin{aligned}
w(\theta) & =\mathcal{J}_{1}\left(\theta_{1}, w\left(\theta_{1}^{-}\right)\right)+w\left(\theta_{1}^{-}\right)+\frac{1}{\Gamma(q)} \int_{\theta_{1}}^{\theta}(\theta-\zeta)^{q-1} g(\zeta) \Delta \zeta \\
& =w_{0}+\mathcal{J}_{1}\left(\theta_{1}, w\left(\theta_{1}^{-}\right)\right)+\frac{1}{\Gamma(q)} \int_{0}^{\theta_{1}}\left(\theta_{1}-\zeta\right)^{q-1} g(\zeta)+\frac{1}{\Gamma(q)} \int_{\theta_{1}}^{\theta}(\theta-\zeta)^{q-1} g(\zeta) \Delta \zeta
\end{aligned}
$$

and so, equation (2.5) holds for $l=1$. Next, we suppose that the equation (2.5) holds for $l=n-1$, then for $\theta \in\left(\theta_{n}, \theta_{n+1}\right]_{\mathbb{T}}$, the initial value problem

$$
\left\{\begin{array}{l}
{ }^{c} \Delta^{q} w(\theta)=g(\theta), \\
w\left(\theta_{n}^{+}\right)-w\left(\theta_{n}^{-}\right)=\partial_{n}\left(\theta_{n}, w\left(\theta_{n}^{-}\right)\right),
\end{array}\right.
$$


has the solution

$$
w(\theta)=w\left(\theta_{n}^{+}\right)+\frac{1}{\Gamma(q)} \int_{\theta_{n}}^{\theta}(\theta-\zeta)^{q-1} g(\zeta) \Delta \zeta, \quad \theta \in\left(\theta_{n}, \theta_{n+1}\right]_{\mathbb{T}} .
$$

Subsequently, we find

$$
\begin{aligned}
w(\theta)= & w_{0}+\frac{1}{\Gamma(q)} \sum_{i=1}^{n-1} \int_{\theta_{i-1}}^{\theta_{i}}\left(\theta_{i}-\zeta\right)^{q-1} g(\zeta) \Delta \zeta+\sum_{i=1}^{n-1} \partial_{i}\left(\theta_{i}, w\left(\theta_{i}^{-}\right)\right)+\mathcal{J}_{n}\left(\theta_{n}, w\left(\theta_{n}^{-}\right)\right) \\
& +\frac{1}{\Gamma(q)} \int_{\theta_{n-1}}^{\theta_{n}}\left(\theta_{n}-\zeta\right)^{q-1} g(\zeta) \Delta \zeta+\frac{1}{\Gamma(q)} \int_{\theta_{n}}^{\theta}(\theta-\zeta)^{q-1} g(\zeta) \Delta \zeta \\
= & w_{0}+\frac{1}{\Gamma(q)} \sum_{i=1}^{n} \int_{\theta_{i-1}}^{\theta_{i}}\left(\theta_{i}-\zeta\right)^{q-1} g(\zeta) \Delta \zeta+\sum_{i=1}^{n} \partial_{i}\left(\theta_{i}, w\left(\theta_{i}^{-}\right)\right)+\frac{1}{\Gamma(q)} \int_{\theta_{n}}^{\theta}(\theta-\zeta)^{q-1} g(\zeta) \Delta \zeta,
\end{aligned}
$$

which means that equation (2.5) is true for $l=n$. Therefore, by the Mathematical Induction, result follows.

To establish the main results of the paper, we require the following assumptions.

(A1): The non-linear function $\mathcal{G}: I \times \mathbb{R} \times \mathbb{R} \rightarrow \mathbb{R}$ is continuous and there exist constants $K_{\mathcal{G}}>0$ and $0<L_{\mathcal{G}}<1$ such that

$$
\left|\mathcal{G}\left(\theta, w_{1}, w_{2}\right)-\mathcal{G}\left(\theta, z_{1}, z_{2}\right)\right| \leq K_{\mathcal{G}}\left|w_{1}-z_{1}\right|+L_{\mathcal{G}}\left|w_{2}-z_{2}\right|, \quad \forall \theta \in I, w_{j}, z_{j} \in \mathbb{R}, j=1,2 .
$$

Also, there exists constants $C_{\mathcal{G}}>0, M_{\mathcal{G}}>0$ and $0<N_{\mathcal{G}}<1$ such that

$$
|\mathcal{G}(\theta, w, z)| \leq C_{\mathcal{G}}+M_{\mathcal{G}}|w|+N_{\mathcal{G}}|z|, \quad \forall \theta \in I, w, z \in \mathbb{R} .
$$

(A2): The functions $\mathcal{J}_{l}(\theta, w)$ are continuous for all $l=1,2, \cdots, p$ and satisfies the following:

(A2a):There exists positive constants $M_{\mathfrak{J}_{l}}, l=1,2, \cdots p$, such that

$$
\left|\mathcal{J}_{l}(\theta, w)\right| \leq M_{\mathfrak{J}_{l}} \forall \theta \in I, w \in \mathbb{R} .
$$

(A2b):There exists positive constants $L_{\mathfrak{J}_{l}}, l=1,2, \cdots, p$, such that

$$
\left|\mathcal{J}_{l}(\theta, w)-\mathcal{J}_{l}(\theta, z)\right| \leq L_{\mathcal{J}_{l}}|w-z|, \forall \theta \in I, w, z \in \mathbb{R} .
$$

(A3): $\frac{M_{\mathcal{G}} T^{q}(p+1)}{\left(1-N_{\mathcal{G}}\right) \Gamma(q+1)}<1$.

\section{Existence and Uniqueness}

Theorem 3.1. If all the assumptions (A1)-(A3) and

$$
\sum_{i=1}^{p} L_{\mathcal{J}_{i}}+\frac{K_{\mathcal{G}} T^{q}(p+1)}{\left(1-L_{\mathcal{G}}\right) \Gamma(q+1)}<1
$$

are satisfied, then the equation (1.1) has a unique solution on I.

Proof: Consider a subset $\Omega \subseteq P C(I, \mathbb{R})$ such that

$$
\Omega=\left\{w \in P C(I, \mathbb{R}):\|w\|_{P C} \leq \beta\right\},
$$


where

$$
\beta=\frac{\left|w_{o}\right|+\sum_{i=1}^{p} M_{\mathfrak{J}_{i}}+\frac{(p+1) C_{\mathcal{G}} T^{q}}{\left(1-N_{\mathcal{G}}\right) \Gamma(q+1)}}{1-\frac{(p+1) M_{\mathcal{G}} T^{q}}{\left(1-N_{\mathcal{G}}\right) \Gamma(q+1)}} .
$$

Now, define an operator $\Pi: \Omega \rightarrow \Omega$ given by

$$
\begin{array}{rlr}
(\Pi w) \theta= & w_{0}+\frac{1}{\Gamma(q)} \int_{0}^{\theta}(\theta-\zeta)^{q-1} \mathcal{G}(\zeta, w(\zeta), v(\zeta)) \Delta \zeta, & \theta \in\left[0, \theta_{1}\right]_{\mathbb{T}} . \\
(\Pi w) \theta= & w_{0}+\frac{1}{\Gamma(q)} \sum_{i=1}^{l} \int_{\theta_{i-1}}^{\theta_{i}}\left(\theta_{i}-\zeta\right)^{q-1} \mathcal{G}(\zeta, w(\zeta), v(\zeta)) \Delta \zeta+\sum_{i=1}^{l} \partial_{i}\left(\theta_{i}, w\left(\theta_{i}^{-}\right)\right) \\
& +\frac{1}{\Gamma(q)} \int_{\theta_{l}}^{\theta}(\theta-\zeta)^{q-1} \mathcal{G}(\zeta, w(\zeta), v(\zeta)) \Delta \zeta, & \theta \in\left(\theta_{l}, \theta_{l+1}\right]_{\mathbb{T}} .
\end{array}
$$

To use the Banach contraction theorem, we need to show that $\Pi: \Omega \rightarrow \Omega$. Now, for $\theta \in\left(\theta_{l}, \theta_{l+1}\right]_{\mathbb{T}}$ and $w \in \Omega$, we have:

$$
\begin{aligned}
|(\Pi w)(\theta)| \leq & \left|w_{0}\right|+\left|\frac{1}{\Gamma(q)} \sum_{i=1}^{l} \int_{\theta_{i-1}}^{\theta_{i}}\left(\theta_{i}-\zeta\right)^{q-1} v(\zeta) \Delta \zeta\right|+\left|\sum_{i=1}^{l} \partial_{i}\left(\theta_{i}, w\left(\theta_{i}^{-}\right)\right)\right| \\
& +\left|\frac{1}{\Gamma(q)} \int_{\theta_{l}}^{\theta}(\theta-\zeta)^{q-1} v(\zeta) \Delta \zeta\right|,
\end{aligned}
$$

where $v \in C(I, \mathbb{R})$ and given by $v(\theta)=\mathcal{G}(\theta, w(\theta), v(\theta))$. Also,

$$
|v(\theta)|=\mid \mathcal{G}\left(\theta, w(\theta), v(\theta)\left|\leq C_{\mathcal{G}}+M_{\mathcal{G}}\right| w\left|+N_{\mathcal{G}}\right| v(\theta) \mid,\right.
$$

which immediately give,

$$
|v(\theta)| \leq \frac{C_{\mathcal{G}}+M_{\mathcal{G}} \beta}{1-N_{\mathcal{G}}} .
$$

Thus, from the inequality (3.4), we have:

$$
|(\Pi w) \theta| \leq\left|w_{0}\right|+\sum_{i=1}^{p} M_{\mathcal{J}_{i}}+\frac{C_{\mathcal{G}}+M_{\mathcal{S}} \beta}{\left(1-N_{\mathcal{G}}\right) \Gamma(q)}\left[\sum_{i=1}^{p} \int_{\theta_{i-1}}^{\theta_{i}}\left(\theta_{i}-\zeta\right)^{q-1} \Delta \zeta+\int_{\theta_{l}}^{\theta}(\theta-\zeta)^{q-1} \Delta \zeta\right] .
$$

Since $(\theta-\zeta)^{q-1}$ is an increasing function, by using Theorem 2.3 we have:

$$
\int_{0}^{\theta}(\theta-\zeta)^{q-1} \Delta \zeta \leq \int_{0}^{\theta}(\theta-\zeta)^{q-1} d s
$$

Consequently,

$$
|(\Pi w) \theta| \leq\left|w_{0}\right|+\sum_{i=1}^{p} M_{\mathcal{J}_{i}}+\frac{C_{\mathcal{G}}+M_{\mathcal{G}} \beta}{\left(1-N_{\mathcal{G}}\right) \Gamma(q)}\left[\sum_{i=1}^{p} \int_{\theta_{i-1}}^{\theta_{i}}\left(\theta_{i}-\zeta\right)^{q-1} d s+\int_{\theta_{l}}^{\theta}(\theta-\zeta)^{q-1} d s\right] .
$$

Hence,

$$
\|\Pi w\|_{P C} \leq\left|w_{0}\right|+\sum_{i=1}^{p} M_{\mathcal{J}_{i}}+\frac{(p+1)\left(C_{\mathcal{G}}+M_{\mathcal{G}} \beta\right) T^{q}}{\left(1-N_{\mathcal{G}}\right) \Gamma(q+1)} .
$$


Also, for $\theta \in\left[0, \theta_{1}\right]_{\mathbb{T}}$ and $w \in \Omega$, one can find that

$$
\|\Pi w\|_{P C} \leq\left|w_{0}\right|+\frac{\left(C_{\mathcal{G}}+M_{\mathcal{G}} \beta\right) T^{q}}{\left(1-N_{\mathcal{G}}\right) \Gamma(q+1)} .
$$

Summarize the above inequalities (3.5) and (3.6), we get:

$$
\|\Pi w\|_{P C} \leq \beta .
$$

Therefore, $\Pi: \Omega \rightarrow \Omega$. For any $w, z \in \Omega, \theta \in\left(\theta_{l}, \theta_{l+1}\right]_{\mathbb{T}}, l=1,2, \cdots, p$, we have:

$$
\begin{aligned}
|(\Pi w)(\theta)-(\Pi z)(\theta)| \leq & \left|\sum_{i=1}^{l} \partial_{i}\left(\theta_{i}, w\left(\theta_{i}^{-}\right)\right)-\partial_{i}\left(\theta_{i}, z\left(\theta_{i}^{-}\right)\right)\right|+\left|\frac{1}{\Gamma(q)} \int_{\theta_{l}}^{\theta}(\theta-\zeta)^{q-1}(v(\zeta)-u(\zeta)) \Delta \zeta\right| \\
& +\frac{1}{\Gamma(q)}\left|\sum_{i=1}^{l} \int_{\theta_{i-1}}^{\theta_{i}}\left(\theta_{i}-\zeta\right)^{q-1}(v(\zeta)-u(\zeta)) \Delta \zeta\right|,
\end{aligned}
$$

where $u \in C(I, \mathbb{R})$ and given by $u(\theta)=\mathcal{G}(\theta, z(\theta), u(\theta))$. Also,

$$
|v(\theta)-u(\theta)| \leq|\mathcal{G}(\theta, w(\theta), v(\theta))-\mathcal{G}(\theta, z(\theta), u(\theta))| \leq K_{\mathcal{G}}|w(\theta)-z(\theta)|+L_{\mathcal{G}}|v(\theta)-u(\theta)|,
$$

which immediately gives

$$
|v(\theta)-u(\theta)| \leq \frac{K_{\mathcal{G}}|w(\theta)-z(\theta)|}{1-L_{\mathcal{G}}} .
$$

Thus, from the inequality (3.8), we get

$$
\begin{aligned}
|(\Pi w)(\theta)-(\Pi z)(\theta)| \leq & \sum_{i=1}^{p} L_{\mathcal{J}_{i}}\left|w\left(\theta_{i}^{-}\right)-z\left(\theta_{i}^{-}\right)\right|+\frac{K_{\mathcal{G}}}{\left(1-L_{\mathcal{G}}\right) \Gamma(q)} \int_{\theta_{l}}^{\theta}(\theta-\zeta)^{q-1}|w(\zeta)-z(\zeta)| \Delta \zeta \\
& +\frac{K_{\mathcal{G}}}{\left(1-L_{\mathcal{G}}\right) \Gamma(q)} \sum_{i=1}^{p} \int_{\theta_{i-1}}^{\theta_{i}}\left(\theta_{i}-\zeta\right)^{q-1}|w(\zeta)-z(\zeta)| \Delta \zeta \\
\leq & \|w-z\|_{P C} \sum_{i=1}^{p} L_{\mathcal{J}_{i}}+\frac{K_{\mathcal{G}}\|w-z\|_{P C}}{\left(1-L_{\mathcal{G}}\right) \Gamma(q)} \int_{\theta_{l}}^{\theta}(\theta-\zeta)^{q-1} \Delta \zeta \\
& +\frac{K_{\mathcal{G}}\|w-z\|_{P C}}{\left(1-L_{\mathcal{G}}\right) \Gamma(q)} \sum_{i=1}^{p} \int_{\theta_{i-1}}^{\theta_{i}}\left(\theta_{i}-\zeta\right)^{q-1} \Delta \zeta \\
\leq & \|w-z\|_{P C} \sum_{i=1}^{p} L_{\mathcal{J}_{i}}+\frac{K_{\mathcal{G}} T^{q}|| w-z \|_{P C}}{\left(1-L_{\mathcal{G}}\right) \Gamma(q+1)}+\frac{p K_{\mathcal{G}} T^{q}\|w-z\|_{P C}}{\left(1-L_{\mathcal{G}}\right) \Gamma(q+1)}
\end{aligned}
$$

Therefore,

$$
\|(\Pi w)-(\Pi z)\|_{P C} \leq\left(\sum_{i=1}^{p} L_{\mathcal{J}_{i}}+\frac{K_{\mathcal{G}} T^{q}(p+1)}{\left(1-L_{\mathcal{G}}\right) \Gamma(q+1)}\right)\|w-z\|_{P C} .
$$

Similarly, for $\theta \in\left[0, \theta_{1}\right]_{\mathbb{T}}$, we can easily obtain

$$
\|(\Pi w)-(\Pi z)\|_{P C} \leq \frac{K_{f} T^{q}}{\left(1-L_{\mathcal{G}}\right) \Gamma(q+1)}\|w-z\|_{P C} .
$$

After summarize the inequalities (3.9) and (3.10), we have the following:

$$
\|(\Pi w)-(\Pi z)\|_{P C} \leq L_{\alpha}\|w-z\|_{P C},
$$


where

$$
L_{\alpha}=\sum_{i=1}^{p} L_{\mathcal{J}_{i}}+\frac{K_{\mathcal{G}} T^{q}(p+1)}{\left(1-L_{\mathcal{G}}\right) \Gamma(q+1)} .
$$

Hence, $\Pi$ is a strict contraction operator. Therefore, be means of Banach contraction theorem, we have a unique fixed point of $\Pi$ which is the solution of equation (1.1).

Next, with the help of fixed point theorem due to Leray Schauder, we will give the sufficient conditions for the existence of at least one solution for the equation (1.1).

Theorem 3.2. If the assumptions (A1) and (A2a) are satisfied, then (1.1) has at least one solution on I, provided there exists a positive constant $\mathcal{K}$ such that

$$
\left|w_{0}\right|+\sum_{i=1}^{p} M_{L_{i}}+\frac{\left(C_{\mathcal{G}}+M_{\mathcal{G}} \mathcal{K}\right) T^{q}(p+1)}{\left(1-N_{\mathcal{G}}\right) \Gamma(q+1)}<\mathcal{K} .
$$

Proof: The proof of this theorem is separated into four steps:

Step 1: Firstly, we prove that the operator defined by equation (3.2) and (3.3) is continuous. Consider a sequence $w_{n}$ such that $w_{n} \rightarrow w$ in $P C(I, \mathbb{R})$. Then for each $\theta \in\left(\theta_{l}, \theta_{l+1}\right]_{\mathbb{T}}, l=1,2, \cdots, p$,

$$
\begin{aligned}
\left|\left(\Pi w_{n}\right)(\theta)-(\Pi w)(\theta)\right| \leq & \left|\sum_{i=1}^{l} \partial_{i}\left(\theta_{i}, w_{n}\left(\theta_{i}^{-}\right)\right)-\partial_{i}\left(\theta_{i}, w\left(\theta_{i}^{-}\right)\right)\right| \\
& +\left|\frac{1}{\Gamma(q)} \int_{\theta_{l}}^{\theta}(\theta-\zeta)^{q-1}\left(v_{n}(\zeta)-v(\zeta)\right) \Delta \zeta\right| \\
& +\frac{1}{\Gamma(q)}\left|\sum_{i=1}^{l} \int_{\theta_{i-1}}^{\theta_{i}}\left(\theta_{i}-\zeta\right)^{q-1}\left(v_{n}(\zeta)-v(\zeta)\right) \Delta \zeta\right|,
\end{aligned}
$$

where $v_{n}, v \in C(I, \mathbb{R})$ and given by

$$
v_{n}(\theta)=\mathcal{G}\left(\theta, w_{n}(\theta), v_{n}(\theta)\right), v(\theta)=\mathcal{G}(\theta, w(\theta), v(\theta)) .
$$

Therefore,

$$
\begin{aligned}
\left|v_{n}-v\right| & =\left|\mathcal{G}\left(\theta, w_{n}, v_{n}\right)-\mathcal{G}(\theta, w, v)\right| \\
& \leq K_{\mathcal{G}}\left|w_{n}-w\right|+L_{\mathcal{G}}\left|v_{n}-v\right| \leq \frac{K_{\mathcal{G}}}{1-L_{\mathcal{G}}}\left|w_{n}-w\right| .
\end{aligned}
$$

Since, $\mathcal{G}(\theta, w, z)$ is continuous, $w_{n} \rightarrow w$ as $n \rightarrow \infty$, hence, from the above inequality we get, $v_{n} \rightarrow v$ as $n \rightarrow \infty$. Also, $\mathcal{J}_{l}(\theta, w(\theta))$ are continues w.r.t. $w$. Therefore by Lebesgue dominated convergence theorem we get:

$$
\left\|\Pi w_{n}-\Pi w\right\|_{P C} \rightarrow 0 \text { as } n \rightarrow \infty .
$$

Similarly, for $\theta \in\left[0, \theta_{1}\right]_{\mathbb{T}}$, we can show that

$$
\left\|\Pi w_{n}-\Pi w\right\|_{P C} \rightarrow 0 \text { as } n \rightarrow \infty .
$$

Thus, $\Pi$ is continuous.

Step 2: The operator $\Pi$ maps bounded sets into bounded sets in $P C(I, \mathbb{R})$. Let $\Omega$ is same as in Theorem 3.1, we have:

$$
\|\Pi w\|_{P C} \leq \beta, \quad \forall w \in \Omega, \theta \in I .
$$


The proof of inequality (3.12) is similar to that of inequality (3.7), hence it is omitted.

Step 3: Let $\zeta_{1}, \zeta_{2} \in\left(\theta_{l}, \theta_{l+1}\right]_{\mathbb{T}}, l=1,2, \cdots, p$, such that $\zeta_{1}<\zeta_{2}$, then we have:

$$
\begin{aligned}
\left|(\Pi w)\left(\zeta_{2}\right)-(\Pi w)\left(\zeta_{1}\right)\right| \leq & \left|\frac{1}{\Gamma(q)} \int_{\theta_{l}}^{\zeta_{1}}\left(\left(\zeta_{2}-\zeta\right)^{q-1}-\left(\zeta_{1}-\zeta\right)^{q-1}\right) v(\zeta) \Delta \zeta\right| \\
& +\left|\frac{1}{\Gamma(q)} \int_{\zeta_{1}}^{\zeta_{2}}\left(\zeta_{2}-\zeta\right)^{q-1} v(\zeta) \Delta \zeta\right| \\
\leq & \left.\frac{C_{\mathcal{G}}+M_{\mathcal{G}}\|w\|_{P C}}{\left(1-N_{\mathcal{G}}\right) \Gamma(q)} \int_{\theta_{l}}^{\zeta_{1}}\left(\zeta_{2}-\zeta\right)^{q-1}-\left(\zeta_{1}-\zeta\right)^{q-1}\right) \Delta \zeta \\
& +\frac{C_{\mathcal{G}}+M_{\mathcal{G}}\|w\|_{P C}}{\left(1-N_{\mathcal{G}}\right) \Gamma(q)} \int_{\zeta_{1}}^{\zeta_{2}}\left(\zeta_{2}-\zeta\right)^{q-1} \Delta \zeta .
\end{aligned}
$$

Since $(\theta-\zeta)^{q-1}$ is continuous, hence $\left|(\Pi w)\left(\zeta_{2}\right)-(\Pi w)\left(\zeta_{1}\right)\right|$ tends to zero as $\zeta_{1} \rightarrow \zeta_{2}$.

Similarly, when $\zeta_{1}, \zeta_{2} \in\left[0, \theta_{1}\right]_{\mathbb{T}}$, we can easily show that

$$
\left|(\Pi w)\left(\zeta_{2}\right)-(\Pi w)\left(\zeta_{1}\right)\right| \rightarrow 0 \text { as } \zeta_{1} \rightarrow \zeta_{2} .
$$

Therefore, collecting the step 1 to step 3 along with the Arzela-Ascoli theorem, we find that $\Pi$ is completely continuous.

Step 4: Let for $\lambda \in[0,1]$, there exists a $w(\theta)$ such that $w(\theta)=\lambda(\Pi w) \theta$. Then for $\theta \in\left(\theta_{l}, \theta_{l+1}\right]_{\mathbb{T}}, l=1,2, \cdots, p$, we have:

$$
\begin{aligned}
|w(\theta)|= & |\lambda \Pi(w) \theta| \\
= & \mid \lambda\left(w_{0}+\frac{1}{\Gamma(q)} \sum_{i=1}^{l} \int_{\theta_{i-1}}^{\theta_{i}}\left(\theta_{i}-\zeta\right)^{q-1} \mathcal{G}(\zeta, w(\zeta), v(\zeta)) \Delta \zeta\right. \\
& \left.+\sum_{i=1}^{l} \partial_{i}\left(\theta_{i}, w\left(\theta_{i}^{-}\right)\right)+\frac{1}{\Gamma(q)} \int_{\theta_{l}}^{\theta}(\theta-\zeta)^{q-1} \mathcal{G}(\zeta, w(\zeta), v(\zeta)) \Delta \zeta\right) \mid \\
\leq & \left|w_{0}\right|+\sum_{i=1}^{p} M_{L_{i}}+\frac{\left(C_{\mathcal{G}}+M_{\mathcal{G}}\|w\|_{P C}\right) T^{q}(p+1)}{\left(1-N_{\mathcal{G}}\right) \Gamma(q+1)} .
\end{aligned}
$$

Thus,

$$
\frac{\|w\|_{P C}}{\left|w_{0}\right|+\sum_{i=1}^{p} M_{L_{i}}+\frac{\left(C_{\mathcal{G}}+M_{\mathcal{G}}\|w\|_{P C}\right) T^{q}(p+1)}{\left(1-N_{\mathcal{G}}\right) \Gamma(q+1)}} \leq 1 .
$$

Similarly, for $\theta \in\left[0, \theta_{1}\right]_{\mathbb{T}}$, we can find that

$$
\frac{\|w\|_{P C}}{\left|w_{0}\right|+\frac{\left(C_{\mathcal{G}}+M_{\mathcal{G}}\|w\|_{P C}\right) T^{q}}{\left(1-N_{\mathcal{G}}\right) \Gamma(q+1)}} \leq 1 .
$$

Now, the condition (3.11) gives a positive number $\mathcal{K}$ such that $\|w\|_{P C} \neq \mathcal{K}$. Let us suppose that $\mathfrak{N}=\{w \in$ $\left.C(I, \mathbb{R}):\|w\|_{P C}<\mathcal{K}\right\}$. The operator $\Pi: \overline{\mathfrak{N}} \rightarrow C(I, \mathbb{R})$ is continuous and completely continuous. Therefore, from the choice of $\mathfrak{N}$, there is no $w \in \partial(\mathfrak{N})$ such that $w=\lambda \Pi w, \lambda \in[0,1]$. Thus, the nonlinear alternative of Leray Schauder type theorem gives a fixed point of $\Pi$, which is the solution of the equation (1.1). 


\section{Stability Analysis}

In this segment, we establish the stability results for the equation (1.1).

For $\epsilon>0, \psi \geq 0$, and nondecresing $\varphi \in P C\left(I, \mathbb{R}^{+}\right)$, consider the following inequalities

$$
\begin{aligned}
& \left\{\begin{array}{l}
\left|{ }^{c} \Delta^{q} z(\theta)-\mathcal{G}\left(\theta, z(\theta),{ }^{c} \Delta^{q} z(\theta)\right)\right| \leq \epsilon, \quad \theta \in I . \\
\left|z\left(\theta_{l}^{+}\right)-z\left(\theta_{l}^{-}\right)-\mathcal{J}_{l}\left(\theta_{l}, z\left(\theta_{l}^{-}\right)\right)\right| \leq \epsilon, \quad l=1,2, \cdots, p .
\end{array}\right. \\
& \left\{\begin{array}{l}
\left|{ }^{c} \Delta^{q} z(\theta)-\mathcal{G}\left(\theta, z(\theta),{ }^{c} \Delta^{q} z(\theta)\right)\right| \leq \varphi(\theta), \quad \theta \in I . \\
\left|z\left(\theta_{l}^{+}\right)-z\left(\theta_{l}^{-}\right)-\mathcal{J}_{l}\left(\theta_{l}, z\left(\theta_{l}^{-}\right)\right)\right| \leq \psi, \quad l=1,2, \cdots, p .
\end{array}\right.
\end{aligned}
$$

and

$$
\left\{\begin{array}{l}
\left|{ }^{c} \Delta^{q} z(\theta)-\mathcal{G}\left(\theta, z(\theta),{ }^{c} \Delta^{q} z(\theta)\right)\right| \leq \epsilon \varphi(\theta), \quad \theta \in I . \\
\left|z\left(\theta_{l}^{+}\right)-z\left(\theta_{l}^{-}\right)-\mathcal{J}_{l}\left(\theta_{l}, z\left(\theta_{l}^{-}\right)\right)\right| \leq \epsilon \psi, \quad l=1,2, \cdots, p .
\end{array}\right.
$$

Definition 4.1. [27] Equation (1.1) is said to be Ulam-Hyer's stable if there exists a positive constant $H_{\left(K_{g}, L_{g}, L_{J_{1}}, q\right)}$ such that for $\epsilon>0$ and for each solution $z$ of inequality (4.13), there exists a unique solution $w$ of equation (1.1) satisfies the following inequality

$$
|z(\theta)-w(\theta)| \leq H_{\left(K_{g}, L_{g}, L_{\mathfrak{I}_{l}}, q\right)} \epsilon, \quad \forall \theta \in I .
$$

Definition 4.2. [27] Equation (1.1) is said to be generalized Ulam-Hyer's stable if there exists $\mathcal{H}_{\left(K_{g}, L_{g}, L_{J_{1}}, q\right)} \in$ $C\left(\mathbb{R}^{+}, \mathbb{R}^{+}\right), \mathcal{H}_{\left(K_{g}, L_{g}, L_{g_{1}}, q\right)}(0)=0$ such that for each solution $z$ of inequalities (4.13), there exists a solution $w$ of equation (1.1) satisfies the following inequality

$$
|z(\theta)-w(\theta)| \leq \mathcal{H}_{\left(K_{g}, L_{g}, L_{J_{l}}, q\right)}(\epsilon), \quad \forall \theta \in I .
$$

Remark 4.3. Definition (4.1) $\Longrightarrow$ Definition (4.2).

Definition 4.4. [27] Equation (1.1) is said to be Ulam-Hyers-Rassias stable with respect to $(\varphi, \psi)$, if there exists $H_{\left(K_{g}, L_{g}, L_{g_{1}}, q, \varphi\right)}$ such that for $\epsilon>0$ and for each solution $z$ of inequality (4.15), there exists a unique solution $w$ of equation (1.1) satisfies the following inequality

$$
|z(\theta)-w(\theta)| \leq H_{\left(K_{\mathcal{g}}, L_{g}, L_{\vartheta_{l}}, q, \varphi\right)} \epsilon(\varphi(\theta), \psi), \quad \forall \theta \in I .
$$

Definition 4.5. [27] Equation (1.1) is said to be generalized Ulam-Hyers-Rassias stable with respect to ( $\varphi, \psi)$, if there exists $H_{\left(K_{g}, L_{g}, L_{I_{l}}, q, \varphi\right)}$ such that for each solution $z$ of inequality (4.14), there exists a unique solution $w$ of equation (1.1) satisfies the following inequality

$$
|z(\theta)-w(\theta)| \leq H_{\left(K_{g}, L_{g}, L_{g_{l}}, q, \varphi\right)}(\varphi(\theta), \psi), \quad \forall \theta \in I .
$$

Remark 4.6. Definition (4.4) $\Longrightarrow$ Definition (4.5).

Remark 4.7. A function $z \in P C^{1}(I, \mathbb{R})$ is a solution of inequality (4.13) if and only if there is $G \in P C(I, \mathbb{R})$ and a sequence $G_{l}, l=1,2, \cdots, p$, such that

(a) $\|G(\theta)\| \leq \epsilon, \forall \theta \in I, \theta \neq \theta_{l}$ and $\left|G_{l}\right| \leq \epsilon, \forall l=1,2, \cdots, p$.

(b) ${ }^{c} \Delta^{q} z(\theta)=\mathcal{G}\left(\theta, z(\theta),{ }^{c} \Delta^{q} z(\theta)\right)+G(\theta), \theta \in I, \theta \neq \theta_{l}$.

(c) $z\left(\theta_{l}^{+}\right)-z\left(\theta_{l}^{-}\right)=\mathcal{J}_{l}\left(\theta_{l}, z\left(\theta_{l}^{-}\right)\right)+G_{l}, l=1,2, \cdots, p$.

One can have the similar remarks for the inequality (4.14) and (4.15). 
Now, by the above Remark 4.7, we have:

$$
\left\{\begin{array}{l}
{ }^{c} \Delta^{q} z(\theta)=\mathcal{G}\left(\theta, z(\theta),{ }^{c} \Delta^{q} z(\theta)\right)+G(\theta), \theta \in I, \theta \neq \theta_{l}, \\
z\left(\theta_{l}^{+}\right)-z\left(\theta_{l}^{-}\right)=\mathcal{J}_{l}\left(\theta_{l}, z\left(\theta_{l}^{-}\right)\right)+G_{l}, l=1,2, \cdots, p .
\end{array}\right.
$$

From Lemma 2.9, one can find that the solution $z$ with $z(0)=w_{0}$ of the above equation is given by

$$
z(\theta)=\left\{\begin{array}{c}
w_{0}+\frac{1}{\Gamma(q)} \int_{0}^{\theta}(\theta-\zeta)^{q-1} u(\zeta) \Delta \zeta+\frac{1}{\Gamma(q)} \int_{0}^{\theta}(\theta-\zeta)^{q-1} G(\zeta) \Delta \zeta, \quad \theta \in\left[0, \theta_{1}\right]_{\mathbb{T}} . \\
w_{0}+\frac{1}{\Gamma(q)} \sum_{i=1}^{l} \int_{\theta_{i-1}}^{\theta_{i}}\left(\theta_{i}-\zeta\right)^{q-1} u(\zeta) \Delta \zeta+\frac{1}{\Gamma(q)} \int_{\theta_{l}}^{\theta}(\theta-\zeta)^{q-1} u(\zeta) \Delta \zeta \\
\quad+\frac{1}{\Gamma(q)} \sum_{i=1}^{l} \int_{\theta_{i-1}}^{\theta_{i}}\left(\theta_{i}-\zeta\right)^{q-1} G(\zeta) \Delta \zeta+\frac{1}{\Gamma(q)} \int_{\theta_{l}}^{\theta}(\theta-\zeta)^{q-1} G(\zeta) \Delta \zeta \\
\quad+\sum_{i=1}^{l}\left[\partial_{i}\left(\theta_{i}, z\left(\theta_{i}^{-}\right)\right)+G_{i}\right], \quad \theta \in\left(\theta_{l}, \theta_{l+1}\right]_{\mathbb{T}}, l=1,2, \cdots, p
\end{array}\right.
$$

where $u \in C(I, \mathbb{R})$ and given by $u(\theta)=\mathcal{G}(\theta, z(\theta), u(\theta))$. Therefore, for $\theta \in\left(\theta_{l}, \theta_{l+1}\right]_{\mathbb{T}}, l=1,2, \cdots, p$, we have:

$$
\begin{aligned}
& \left|z(\theta)-w_{0}-\frac{1}{\Gamma(q)} \sum_{i=1}^{l} \int_{\theta_{i-1}}^{\theta_{i}}\left(\theta_{i}-\zeta\right)^{q-1} u(\zeta) \Delta \zeta-\frac{1}{\Gamma(q)} \int_{\theta_{l}}^{\theta}(\theta-\zeta)^{q-1} u(\zeta) \Delta \zeta-\sum_{i=1}^{l} \partial_{i}\left(\theta_{i}, z\left(\theta_{i}^{-}\right)\right)\right| \\
& \quad \leq M \epsilon .
\end{aligned}
$$

Also, for $\theta \in\left[0, \theta_{1}\right]_{\mathbb{T}}$, we get

$$
\left|z(\theta)-w_{0}-\frac{1}{\Gamma(q)} \int_{0}^{\theta}(\theta-\zeta)^{q-1} u(\zeta) \Delta \zeta\right| \leq M^{\star} \epsilon,
$$

where,

$$
M=\frac{(p+1) T^{q}}{\Gamma(q+1)}+p \text { and } M^{\star}=\frac{T^{q}}{\Gamma(q+1)} .
$$

Our next theorem gives sufficient condition under which the equation (1.1) is Hyer-Ulam's stability.

Theorem 4.8. If the inequality (3.1) and the assumptions (A1)-(A2) are satisfied, then the equation (1.1) is Hyer-Ulam's stable.

Proof: Let $z \in P C^{1}(I, \mathbb{R})$ be the solution of inequality (4.13) and $w \in P C(I, \mathbb{R})$ be unique solution of the equation (1.1). Therefore, by Lemma 2.9, we have:

$$
w(\theta)= \begin{cases}w_{0}+\frac{1}{\Gamma(q)} \int_{0}^{\theta}(\theta-\zeta)^{q-1} v(\zeta) \Delta \zeta, & \theta \in\left[0, \theta_{1}\right]_{\mathbb{T}}, \\ w_{0}+\frac{1}{\Gamma(q)} \sum_{i=1}^{l} \int_{\theta_{i-1}}^{\theta_{i}}\left(\theta_{i}-\zeta\right)^{q-1} v(\zeta) \Delta \zeta+\frac{1}{\Gamma(q)} \int_{\theta_{l}}^{\theta}(\theta-\zeta)^{q-1} v(\zeta) \Delta \zeta & \\ \quad+\sum_{i=1}^{l} \partial_{i}\left(\theta_{i}, w\left(\theta_{i}^{-}\right)\right), & \theta \in\left(\theta_{l}, \theta_{l+1}\right]_{\mathbb{T}},\end{cases}
$$


where $v \in C(I, \mathbb{R})$ and given by $v(\theta)=\mathcal{G}(\theta, w(\theta), v(\theta))$. For $\theta \in\left(\theta_{l}, \theta_{l+1}\right]_{\mathbb{T}}, l=1,2, \cdots, p$, we can easily find that

$$
\begin{aligned}
|z(\theta)-w(\theta)| \leq & \mid z(\theta)-w_{0}-\frac{1}{\Gamma(q)} \sum_{i=1}^{l} \int_{\theta_{i-1}}^{\theta_{i}}\left(\theta_{i}-\zeta\right)^{q-1} v(\zeta) \Delta \zeta-\frac{1}{\Gamma(q)} \int_{\theta_{l}}^{\theta}(\theta-\zeta)^{q-1} v(\zeta) \Delta \zeta \\
& -\sum_{i=1}^{l} \partial_{i}\left(\theta_{i}, w\left(\theta_{i}^{-}\right)\right) \mid \\
\leq & \left|\frac{1}{\Gamma(q)} \sum_{i=1}^{l} \int_{\theta_{i-1}}^{\theta_{i}}\left(\theta_{i}-\zeta\right)^{q-1}(u(\zeta)-v(\zeta)) \Delta \zeta\right|+\left|\sum_{i=1}^{l} \partial_{i}\left(\theta_{i}, z\left(\theta_{i}^{-}\right)\right)-\partial_{i}\left(\theta_{i}, w\left(\theta_{i}^{-}\right)\right)\right| \\
& +\left|\frac{1}{\Gamma(q)} \int_{\theta_{l}}^{\theta}(\theta-\zeta)^{q-1}(u(\zeta)-v(\zeta)) \Delta \zeta\right|+\epsilon M .
\end{aligned}
$$

Subsequently, we get

$$
\|z-w\|_{P C} \leq \epsilon M+\left[\sum_{i=1}^{p} L_{\mathcal{J}_{i}}+\frac{K_{\mathcal{G}} T^{q}(p+1)}{\left(1-L_{\mathcal{G}}\right) \Gamma(q+1)}\right]\|z-w\|_{P C},
$$

which immediately gives

$$
\|z-w\|_{P C} \leq \frac{\epsilon M}{1-L_{\alpha}} .
$$

Similarly, for $\theta \in\left[0, \theta_{1}\right]_{\mathbb{T}}$,

$$
\|z-w\|_{P C} \leq \frac{\epsilon M^{\star}}{1-K^{\star}} .
$$

From the inequalities (4.17) and (4.18), we get

$$
\|z-w\|_{P C} \leq \epsilon\left[\frac{M}{1-L_{\alpha}}+\frac{M^{\star}}{1-K^{\star}}\right] .
$$

Hence,

$$
\|z-w\|_{P C} \leq H_{\left(K_{\mathcal{S}}, L_{9}, L_{g_{l}}, q\right)} \epsilon, \quad \theta \in I,
$$

where $H_{\left(K_{g}, L_{g}, L_{J_{l}}, q\right)}=\left[\frac{M}{1-L_{\alpha}}+\frac{M^{\star}}{1-K^{\star}}\right]$. Thus, equation (1.1) is Ulam-Hyer's stable. Moreover, if we put $\mathcal{H}_{\left(K_{\mathcal{S}}, L_{\mathcal{S}}, L_{\mathfrak{I}_{l}}, q\right)}(\epsilon)=H_{\left(K_{\mathcal{S}}, L_{\mathcal{S}}, L_{\jmath_{l}}, q\right)} \epsilon, \mathcal{H}_{\left(K_{\mathcal{S}}, L_{\mathcal{S}}, L_{\mathfrak{I}_{l}}, q\right)}(0)=0$, then equation (1.1) is generalized Ulam-Hyer's stable.

(A4): There exists a $\lambda_{\varphi}>0$ such that ${ }^{\Delta} I^{q} \varphi(\theta) \leq \lambda_{\varphi} \varphi(\theta), \forall \theta \in I$.

Theorem 4.9. If the assumptions (A1), (A2), (A4) and the inequality (3.1) are satisfied, then the equation (1.1) is Ulam-Hyers-Rassias stable.

Proof: Let $z \in P C^{1}(I, \mathbb{R})$ be solution of inequality (4.15) and $w \in P C(I, \mathbb{R})$ be the unique solution of the equation (1.1). Therefore, by Lemma 2.9, we have:

$$
w(\theta)= \begin{cases}w_{0}+\frac{1}{\Gamma(q)} \int_{0}^{\theta}(\theta-\zeta)^{q-1} v(\zeta) \Delta \zeta, & \theta \in\left[0, \theta_{1}\right]_{\mathbb{T}} . \\ w_{0}+\frac{1}{\Gamma(q)} \sum_{i=1}^{l} \int_{\theta_{i-1}}^{\theta_{i}}\left(\theta_{i}-\zeta\right)^{q-1} v(\zeta) \Delta \zeta+\frac{1}{\Gamma(q)} \int_{\theta_{l}}^{\theta}(\theta-\zeta)^{q-1} v(\zeta) \Delta \zeta & \\ \quad+\sum_{i=1}^{l} \partial_{i}\left(\theta_{i}, w\left(\theta_{i}^{-}\right)\right), & \theta \in\left(\theta_{l}, \theta_{l+1}\right]_{\mathbb{T}},\end{cases}
$$


where $v \in C(I, \mathbb{R})$ and given by $v(\theta)=\mathcal{G}(\theta, w(\theta), v(\theta))$. For $\theta \in\left(\theta_{l}, \theta_{l+1}\right]_{\mathbb{T}}, l=1,2, \cdots, p$, we can easily find that

$$
\begin{aligned}
|z(\theta)-w(\theta)| \leq & \mid z(\theta)-w_{0}-\frac{1}{\Gamma(q)} \sum_{i=1}^{l} \int_{\theta_{i-1}}^{\theta_{i}}\left(\theta_{i}-\zeta\right)^{q-1} v(\zeta) \Delta \zeta-\frac{1}{\Gamma(q)} \int_{\theta_{l}}^{\theta}(\theta-\zeta)^{q-1} v(\zeta) \Delta \zeta \\
& -\sum_{i=1}^{l} \partial_{i}\left(\theta_{i}, w\left(\theta_{i}^{-}\right)\right) \mid \\
\leq & \frac{1}{\Gamma(q)} \sum_{i=1}^{l} \int_{\theta_{i-1}}^{\theta_{i}}\left(\theta_{i}-\zeta\right)^{q-1}(u(\zeta)-v(\zeta)) \Delta \zeta|+| \sum_{i=1}^{l} \partial_{i}\left(\theta_{i}, z\left(\theta_{i}^{-}\right)\right)-\partial_{i}\left(\theta_{i}, w\left(\theta_{i}^{-}\right)\right) \mid \\
& +\left|\frac{1}{\Gamma(q)} \int_{\theta_{l}}^{\theta}(\theta-\zeta)^{q-1}(u(\zeta)-v(\zeta)) \Delta \zeta\right|+\epsilon\left(p \psi+(p+1) \lambda_{\varphi} \varphi(\theta)\right) .
\end{aligned}
$$

Subsequently, we get

$$
\|z-w\|_{P C} \leq \epsilon(\varphi(\theta)+\psi)\left(\lambda_{\varphi}(p+1)+p\right)+\left[\sum_{i=1}^{p} L_{\mathcal{J}_{i}}+\frac{K_{\mathcal{G}} T^{q}(p+1)}{\left(1-L_{\mathcal{G}}\right) \Gamma(q+1)}\right]\|z-w\|_{P C},
$$

which immediately gives

$$
\|z-w\|_{P C} \leq \frac{\epsilon(\varphi(\theta)+\psi)\left(\lambda_{\varphi}(p+1)+p\right)}{1-L_{\alpha}}
$$

Similarly, for $\theta \in\left[0, \theta_{1}\right]_{\mathbb{T}}$,

$$
\|z-w\|_{P C} \leq \frac{\epsilon \lambda_{\varphi} \varphi(\theta)}{1-K^{\star}} .
$$

From the inequalities (4.19) and (4.20), we get

$$
\|z-w\|_{P C} \leq \epsilon(\varphi(\theta)+\psi)\left[\frac{\lambda_{\varphi}(p+1)+p}{1-L_{\alpha}}+\frac{\lambda_{\varphi}}{1-K^{\star}}\right] .
$$

Hence,

$$
\|z-w\|_{P C} \leq H_{\left(K_{g}, L_{9}, L_{g_{l}}, q, \varphi\right)} \epsilon(\varphi(\theta)+\psi), \quad \theta \in I,
$$

where $H_{\left(K_{S}, L_{g}, L_{g_{l}}, q, \varphi\right)}=\left[\frac{\lambda_{\varphi}(p+1)+p}{1-L_{\alpha}}+\frac{\lambda_{\varphi}}{1-K^{\star}}\right]$. Thus, equation (1.1) is Ulam-Hyers-Rassias stable.

\section{Non-local problem}

In this section, we consider the equation (1.1) with nonlocal effects of the form:

$$
\begin{aligned}
{ }^{c} \Delta^{q} w(\theta) & =\mathcal{G}\left(\theta, w(\theta),{ }^{c} \Delta^{q} w(\theta)\right), \quad \theta \in I, \quad \theta \neq \theta_{l}, \\
w\left(\theta_{l}^{+}\right) & -w\left(\theta_{l}^{-}\right)=\mathcal{J}_{l}\left(\theta_{l}, w\left(\theta_{l}^{-}\right)\right), \quad l=1,2, \cdots, p, \\
w(0) & =\xi(w)+w_{0} .
\end{aligned}
$$

Definition 5.1. A function $w(\theta) \in P C(I, \mathbb{R})$ is called a solution of the nonlocal problem (5.1), if for each $l=$ $1,2, \cdots, p$, it satisfies the equation ${ }^{c} \Delta^{q} w(\theta)=\mathcal{G}\left(\theta, w(\theta),{ }^{c} \Delta^{q} w(\theta)\right)$ a.e on I and the conditions $w\left(\theta_{l}^{+}\right)-w\left(\theta_{l}^{-}\right)=$ $\partial_{l}\left(\theta_{l}, w\left(\theta_{l}^{-}\right)\right), w(0)=\xi(w)+w_{0}$. 
Lemma 5.2. Let $g: I \rightarrow \mathbb{R}$ be a right dense continuous function. Then for any $l=1,2, \cdots, p$, the solution of problem

$$
\begin{aligned}
{ }^{c} \Delta^{q} w(\theta) & =g(\theta), \quad \theta \in I, \quad \theta \neq \theta_{l}, \\
w\left(\theta_{l}^{+}\right) & -w\left(\theta_{l}^{-}\right)=\mathcal{J}_{l}\left(\theta_{l}, w\left(\theta_{l}^{-}\right)\right), l=1,2, \cdots, p, \\
w(0) & =\xi(w)+w_{0},
\end{aligned}
$$

is given by the following integral equation

$$
w(\theta)=\left\{\begin{array}{cc}
w_{0}+\xi(w)+\frac{1}{\Gamma(q)} \int_{0}^{\theta}(\theta-\zeta)^{q-1} g(\zeta) \Delta \zeta, & \theta \in\left[0, \theta_{1}\right]_{\mathbb{T}} . \\
w_{0}+\xi(w)+\frac{1}{\Gamma(q)} \sum_{i=1}^{l} \int_{\theta_{i-1}}^{\theta_{i}}\left(\theta_{i}-\zeta\right)^{q-1} g(\zeta) \Delta \zeta+\frac{1}{\Gamma(q)} \int_{\theta_{l}}^{\theta}(\theta-\zeta)^{q-1} g(\zeta) \Delta \zeta \\
\quad+\sum_{i=1}^{l} \partial_{i}\left(\theta_{i}, w\left(\theta_{i}^{-}\right)\right), & \theta \in\left(\theta_{l}, \theta_{l+1}\right]_{\mathbb{T}} .
\end{array}\right.
$$

Proof: The proof of this Lemma is the consequence of Lemma 2.5. Hence, we omitted.

Further, to prove our results for the equation (5.1), we need some more assumptions.

(A5):The function $\xi: C(I, \mathbb{R}) \rightarrow \mathbb{R}$ is a continuous function and there exists positive constants $M_{\xi}, L_{\xi}$ such that

$$
|\xi(w)| \leq M_{\xi}, \quad|\xi(w)-\xi(z)| \leq L_{\xi}|w-z|, \quad \forall \theta \in I \text { and } w, z \in \mathbb{R} .
$$

Theorem 5.3. If the assumptions (A1)-(A3) and (A5) with

$$
\sum_{i=1}^{p} L_{\mathfrak{J}_{i}}+L_{\xi}+\frac{K_{\mathcal{G}} T^{q}(p+1)}{\left(1-L_{\mathcal{G}}\right) \Gamma(q+1)}<1,
$$

are satisfied, then equation (5.1) has a unique solution on $I$.

Proof: We can proof this theorem by adopting the strategies of Theorem (3.1). Henceforth, we omitted.

\section{Examples}

Example 6.1. Consider the following implicit equation with impulses on $\mathbb{T},\left(0, \frac{1}{4}, 1 \in \mathbb{T}\right)$

$$
\begin{aligned}
{ }^{c} \Delta^{q} w(\theta) & =\frac{\left.3+|w(\theta)|+\mid{ }^{c} \Delta^{q} w(\theta)\right) \mid}{\left.40 e^{\theta^{2}+3}\left(1+|w(\theta)|+\mid{ }^{c} \Delta^{q} w(\theta)\right) \mid\right)}, \quad \theta \in I=[0,1]_{\mathbb{T}}, \theta \neq \frac{1}{4}, w \in \mathbb{R}, \\
w\left(\theta_{1}^{+}\right) & -w\left(\theta_{1}^{-}\right)=\frac{1+\theta_{1}^{-} \sin \left(w\left(\theta_{1}^{-}\right)\right)}{5}, \quad \theta_{1}=\frac{1}{4}, \\
w(0) & =1 .
\end{aligned}
$$

Here,

$$
\mathcal{G}(\theta, w, v)=\frac{3+|w(\theta)|+|v(\theta)|}{40 e^{\theta^{2}+3}(1+|w(\theta)|+|v(\theta)|)}, \theta \in I, w \in \mathbb{R}
$$

and

$$
J_{1}(\theta, w)=\frac{1+\theta \sin (w(\theta))}{5}, \theta=\theta_{1}, w \in \mathbb{R} .
$$

Then, $\forall \theta \in I, w, z, v, u \in \mathbb{R}$, we have:

$$
\begin{aligned}
& |\mathcal{G}(\theta, w, v)| \leq \frac{3+|w(\theta)|+\left|{ }^{c} \Delta^{q} w(\theta)\right|}{40 e^{3}},|\mathcal{G}(\theta, w, v)-\mathcal{G}(\theta, z, u)| \leq \frac{1}{40 e^{3}}|w-z|+\frac{1}{40 e^{3}}|v-u|, \\
& \left|\mathcal{J}_{1}(\theta, w)-\mathcal{J}_{1}(\theta, z)\right| \leq \frac{1}{5}|w-z| .
\end{aligned}
$$


Hence, the assumptions (A1)-(A3) are satisfied with $C_{\mathcal{G}}=\frac{3}{40 e^{3}}, M_{\mathcal{G}}=\frac{1}{40 e^{3}}, N_{\mathcal{G}}=\frac{1}{40 e^{3}}, K_{\mathcal{G}}=\frac{1}{40 e^{3}}, L_{\mathcal{G}}=$ $\frac{1}{40 e^{3}}, M_{\mathfrak{J}_{1}}=\frac{2}{5}, L_{\mathfrak{J}_{1}}=\frac{1}{5}$. Also, for $p=1, T=1$, the condition

$$
\sum_{i=1}^{p} L_{\mathfrak{J}_{i}}+\frac{K_{\mathcal{G}} T^{q}(p+1)}{\left(1-L_{\mathcal{G}}\right) \Gamma(q+1)}=\frac{1}{5}+\frac{2.492 \times 10^{-3}}{\Gamma(q+1)}<1
$$

is true for all value of $q$. Thus, the conditions of the Theorem 3.1 and Theorem 4.8 are satisfied. Therefore, equation (6.1) has a Ulam Hyer's stable solution which is unique.

Example 6.2. Consider the following implicit problem with impulses and nonlocal condition on the general time scale $\mathbb{T},\left(0, \frac{1}{3}, 1 \in \mathbb{T}\right)$

$$
\begin{aligned}
{ }^{c} \Delta^{q} w(\theta) & =\frac{\left.\sin \theta\left(3+|w(\theta)|+\mid{ }^{c} \Delta^{q} w(\theta)\right) \mid\right)}{\left.25 e^{\theta^{2}+5}\left(1+|w(\theta)|+\mid{ }^{c} \Delta^{q} w(\theta)\right) \mid\right)}, \quad \theta \in I=[0,1]_{\mathbb{T}}, \theta \neq \frac{1}{3}, w \in \mathbb{R}, \\
w\left(\theta_{1}^{+}\right) & -w\left(\theta_{1}^{-}\right)=\frac{1+\theta_{1}^{-} \sin \left(w\left(\theta_{1}^{-}\right)\right)}{5}, \quad \theta_{1}=\frac{1}{3}, \\
w(0) & =1+\frac{1+\sin w}{10} .
\end{aligned}
$$

Here,

$$
\begin{gathered}
\mathcal{G}(\theta, w, v)=\frac{\sin \theta(3+|w(\theta)|+|v(\theta)|)}{25 e^{\theta^{2}+5}(1+|w(\theta)|+|v(\theta)|)}, \theta \in I, w \in \mathbb{R}, \\
J_{1}(\theta, w)=\frac{1+\theta \sin (w(\theta))}{5}, \theta=\theta_{1}, w \in \mathbb{R} \quad \text { and } \xi(w)=\frac{1+\sin w}{10}, w \in \mathbb{R} .
\end{gathered}
$$

Then, $\forall \theta \in I, w, z, v, u \in \mathbb{R}$, we have:

$$
\begin{aligned}
& |\mathcal{G}(\theta, w, v)| \leq \frac{3+|w(\theta)|+|v(\theta)|}{25 e^{5}},|\mathcal{G}(\theta, w, v)-\mathcal{G}(\theta, z, u)| \leq \frac{1}{25 e^{5}}|w-z|+\frac{1}{25 e^{5}}|v-u|, \\
& \left|\mathcal{J}_{1}(\theta, w)-\mathcal{J}_{1}(\theta, z)\right| \leq \frac{1}{5}|w-z|,\left|\xi(w)-\xi(z) \leq \frac{1}{10}\right| w-z|,| \xi(w) \mid \leq \frac{1}{5} .
\end{aligned}
$$

Thus, the assumptions (A1)-(A4) are satisfied. Also, for $T=1, p=1$ the condition

$$
\sum_{i=1}^{p} L_{\mathcal{J}_{i}}+\frac{K_{\mathcal{G}} T^{q}(p+1)}{\left(1-L_{\mathcal{G}}\right) \Gamma(q+1)}+L_{\xi}=\frac{3}{10}+\frac{5.3918 \times 10^{-4}}{\Gamma(q+1)}<1,
$$

is true for all value of $q$. Hence, the conditions of the Theorem 5.3 and Theorem 4.8 are satisfied. Therefore, equation (6.2) has a unique Ulam Hyer's stable solution.

Acknowledgement: We are very thankful to the anonymous reviewer and editor for their constructive comments and suggestions which help us to improve the manuscript. The research of the first author "Vipin Kumar" is supported by the University Grants Commission (UGC) of India under the research fellowship number 2121540900, Ref. no. 20/12/2015 (ii) EU-V.

\section{References}

[1] Diethelm, K., Freed, A.D., On the solution of nonlinear fractional-order differential equations used in the modeling of viscoplasticity. Scientific Computing in Chemical Engineering II. Springer, Berlin, Heidelberg, (1999), 217-224.

[2] Glöckle, W.G., Nonnenmacher, T.F., A fractional calculus approach to self-similar protein dynamics. Biophysical Journal, 68(1) (1995), 46-53.

[3] Zhang, S., Existence of solution for a boundary value problem of fractional order. Acta Mathematica Scientia, 26(2) (2006), 220-228. 
[4] Benchohra, M., Graef, J. R., Hamani, S., Existence results for boundary value problems with non-linear fractional differential equations. Applicable Analysis, 87(7) (2008), 851-863.

[5] Wang, T., Feng, X., Existence and uniqueness of fractional differential equations with integral boundary conditions. The Journal of Nonlinear Sciences and its Applications 1(4) (2008), 206-212.

[6] Ahmad, B., Nieto, J.J., Existence results for nonlinear boundary value problems of fractional integrodifferential equations with integral boundary conditions. Boundary Value Problems, 2009(1) (2009), 708576.

[7] Ahmad, B., Sivasundaram, S., Existence results for nonlinear impulsive hybrid boundary value problems involving fractional differential equations. Nonlinear Analysis: Hybrid Systems, 3(3) (2009), 251-258.

[8] Liu, X., Jia, M., Baofeng, Wu., Existence and uniqueness of solution for fractional differential equations with integral boundary conditions. Electronic Journal of Qualitative Theory of Differential Equations, 2009(69) (2009), 1-10.

[9] Agarwal, R. P., Benchohra, M., Hamani,S., A survey on existence results for boundary value problems of nonlinear fractional differential equations and inclusions. Acta Applicandae Mathematicae, 109(3) (2010), 973-1033.

[10] Benchohra, M., Ouaar, F., Existence results for nonlinear fractional differential equations with integral boundary conditions. Bulletin of Mathematical Analysis and Applications, 2(2) (2010), 7-15.

[11] Lakshmikantham, V., Bainov, D.D., Simeonov, P.S., Theory of impulsive differential equations. Volume 6, World scientific, 1989.

[12] Benchohra, M., Henderson, J., Ntouyas, S.K., Impulsive Differential Equations and Inclusions, Hindawi Publishing Corporation, Volume 2, New York, 2006.

[13] Hilger,S., EinMakettenkalkul mit Anwendung auf Zentrumsmannigfaltigkeiten (Ph.D thesis), Universitt Wurzburg, 1988.

[14] Naidu, D., Singular perturbations and time scales in control theory and applications: an overview. Dynamics of Continuous Discrete and Impulsive Systems Series B, 9 (2002), 233-278.

[15] Zhuang, K., Periodic solutions for a stage-structure ecological model on time scales. Electronic Journal of Differential Equations, 2007 (2007), 1-7.

[16] Ferhan, A. M., Biles, D. C., Lebedinsky, A., An application of time scales to economics. Mathematical and Computer Modelling, 43(7) (2006), 718-726.

[17] Ferhan, A. M., Uysal, F., A production-inventory model of HMMS on time scales. Applied Mathematics Letters, 21(3) (2008), 236-243.

[18] Bohner, M., Peterson, A., Dynamic Equations on Time Scales. Birkhäuser, Basel, 2001.

[19] Bohner, M., Peterson, A., eds.: Advances in Dynamic Equations on Time Scales. Birkhäuser, Boston, MA, 2003.

[20] Agarwal, R. P., Bohner, M., Basic calculus on time scales and some of its applications, Results in Mathematics, 35 (1999), 3-22.

[21] Liu, H., Xiang,X., A class of the first order impulsive dynamic equations on time scales, Nonlinear Analysis: Theory, Methods and Applications, 69(9) (2008), 2803-2811.

[22] Kumar, V., Malik, M., Existence and stability of fractional integro differential equation with non-instantaneous integrable impulses and periodic boundary condition on time scales, Journal of King Saud University - Science (2018), doi: https://doi.org/10.1016/j.jksus.2018.10.011

[23] Ahmadkhanlu, A., Jahanshahi,M., On the existence and uniqueness of solution of initial value problem for fractional order differential equations on time scales. Bulletin of the Iranian Mathematical Society, 38(1) (2012), 241-252.

[24] Benkhettou, N., Hammoudi, A., Torres, D.F.M., Existence and uniqueness of solution for a fractional Riemann-Liouville initial value problem on time scales. Journal of King Saud University-Science, 28 (2016), 87-92.

[25] Yan, R.A., Sun, S.R., Han, Z.L., Existence of solutions of boundary value problems for Caputo fractional differential equations on time scales. Bulletin of the Iranian Mathematical Society, 42(2) (2016), 247-262.

[26] Agarwal, R.P., Benchohra, M., O’Regan, D., Ouahab, A., Second order impulsive dynamic equations on time scales. Functional Differential Equations, 11(3-4) (2004), 223-234.

[27] Wang, J.R., Feĉkan, M., Zhou, Y., Ulam's type stability of impulsive ordinary differential equations. Journal of Mathematical Analysis and Applications, 395(1) (2012), 258-264.

[28] Zada, A., Faisal, S., Li, Y., On the Hyers-Ulam stability of first-order impulsive delay differential equations. Journal of Function Spaces, 2016 (2016).

[29] Haq, F., Shah, K., Rahman, G., Shahzad, M., Hyers-Ulam stability to a class of fractional differential equations with boundary conditions. International Journal of Applied and Computational Mathematics, 3(1) (2017), 1135-1147.

[30] Shen, Y., The Ulam stability of first order linear dynamic equations on time scales. Results in Mathematics, 72(4) (2017), 1881-1895.

[31] András, S., Mészáros, A.R., Ulam-Hyers stability of dynamic equations on time scales via Picard operators. Applied Mathematics and Computation, 219(9) (2013), 4853-4864. 